\title{
Frequency stabilization of an external-cavity diode laser
}

\author{
W. David Lee and Joe C. Campbell \\ Microelectronics Research Center, Department of Electrical and Computer Engineering. The University \\ of Texas at Austin, Austin, Texas 78712 \\ R. J. Brecha and H. J. Kimble \\ Norman Bridge Laboratory of Physics 12-33. California Institute of Technology, Pasadena, California \\ 91125
}

(Received 18 June 1990; accepted for publication 27 August 1990)

Using a hybrid optical/electronic technique, an external-cavity diode laser was frequency stabilized with respect to the sub-Doppler spectrum of cesium vapor. Laser linewidths of 65 $\mathrm{kHz}$ and frequency stabilities of $\pm 10 \mathrm{kHz}$ were obtained.

Linewidth and frecuency stability of a semiconductor laser are important parameters for coherent optical communications. Typically, AlGaAs semiconductor lasers have linewidths of tens of megahertz and their frequency is quite sensitive to changes in temperature and injection current. A variety of electronic frequency stabilization schemes have been developed in order to compensate this sensitivity. "Narrow linewidth have been achieved with external-cavity lasers using mirrors ${ }^{2}$ and diffraction gratings $^{3,4}$ as optical feedback elements. A novel externalcavity laser consisting of a laser coupled to a separate confocal Fabry-Perot resonator yields not only reduced linewidths bat also provides a convenient mechanism for tuning the laser. 5,6 The diode laser optically "self-locks" to a longitudinal mode of the confocal cavity and experiences a reduction in Inewidth. The laser frequency may be tuned by changing the mirror separation of the external resonator. In this letter, we demonstrate frequency stabilization of such an external-cavity laser with respect to the Doppler-free absorpiion spectrum of cesium.

Figure 1 is a schematic of the external-cavity laser. The Fabry-Perot resonator's mirrors are identical and are separated by their radius of curvature $(10 \mathrm{~cm})$. The free spectral range is $750 \mathrm{MH} z$ with a finesse of 60 . The resonator is aligned so that the ight from the laser (Hitachi HLP1400) enters mirror $M_{1}$ at a point that coincides with the center of curvature of mirror $M_{2}$. When the laser frequency is resonant with a longitudinal mode of the Fabry-Perot cavity, a standing wave is formed and a portion of the intracavity feld returns to the laser. The Fabry-Perot serves as an optical feedback element with a tunable, narrow reflectivity spectrum.

The frequency-locking effect resulting from the optical feedback is illustrated in Fig. 2. The Iaser's temperature and injection current were adjusted so that its wavelength coincided with the $D_{2}$ line of cesium. This figure was obtained by diverting part of the laser light through a cesium vapor cell and measuring the transmission with a photodetector. Absorption of the laser light by cesium vapor was recorded as the laser frequency was scanned using the injection current. When the laser frequency did not match a longitudinal mode of the Fabry-Perot cavity the laser frequency scanted continuously with injection current. As the laser frequency neared a longitudinal cavity mode, optical feedback caused the laser frequency to lock to that mode. Frequency locking to the cavity produced a flat region in the absorption spectrum since the laser frequency was held approximately constant by the optical feedback. In this region changes in injection current were largely ignored by the laser frequency.

The width of this fat region, referred to as the "locking bandwidth", can be as large as $500 \mathrm{MHZ} z$ and depends on the intensity of the optical feedback. If nuctuations in injection current and temperature are small, the laser will remain locked to the confocal cavity. An estimate of the

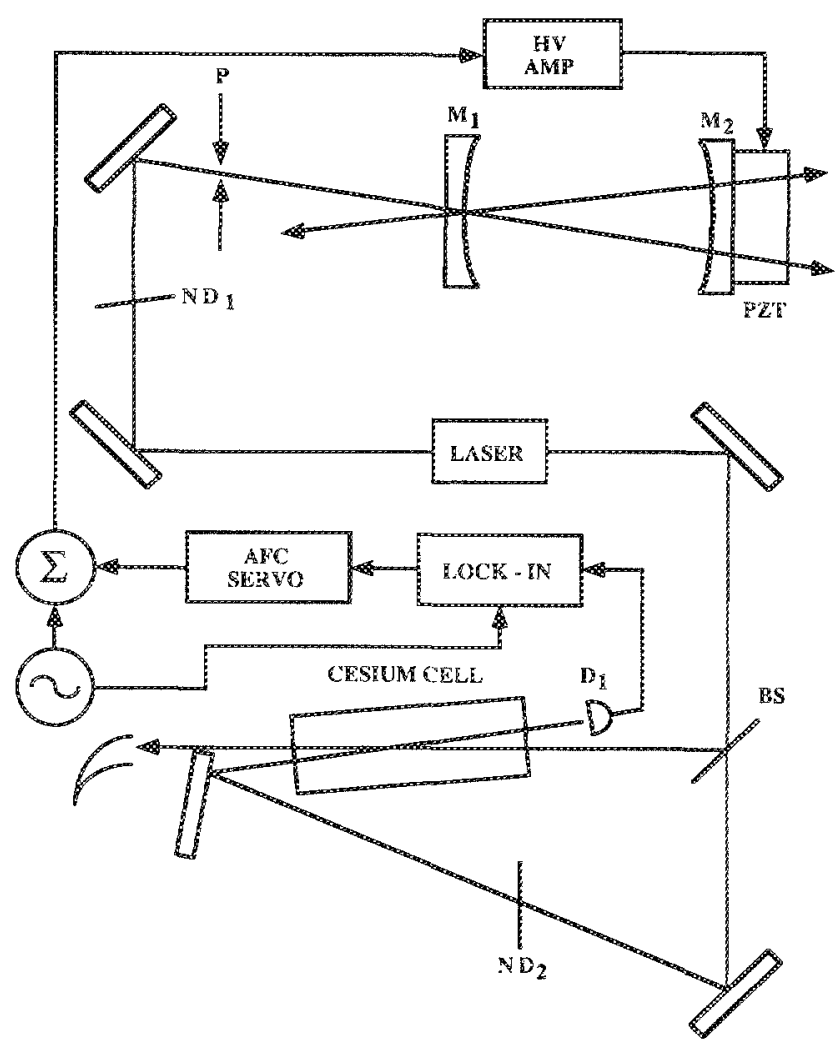

FIG. 1. Schematic of external-cavity laser. The laser's beam is directed to the confocal Fabry-Perot cavity formed by mirrors $M_{1}$ and $M_{2}$. A neutral density filter (ND $)$ is used to adjust the level of fecdback while a pinhole $(P)$ blocks stray reffertions from the cavity. Saturation spectroscopy is used to obtain a sub-Doppler absorption spectrum and an error signal for the automatic frequency control hoop (AFC). The output of the AFC servo is amplified and drives a piezoelectric translator (PZT) in order to stabilize the confocal cavity and therefore the laser frequency. 


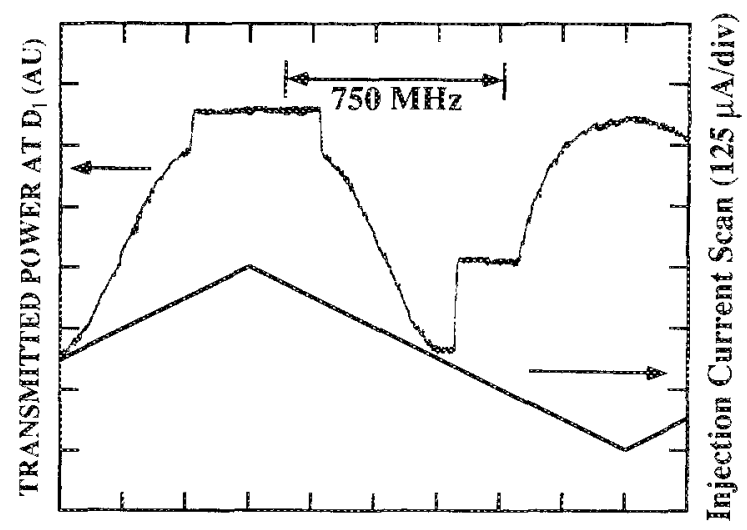

FIG. 2. Effect of optical feedback. Absorption of laser light by cesium vapor is recorded as the injection current is scanned up and down $(\Delta i / i$ $\left.=3 \times 10^{-3}\right)$. This figure shows the locking effect of ontical feedback. The spectrum exhibits a fat region where the absorption remains constant (the laser frequency remains constant) despite the continuous linear in jection current scan ( $20 \mathrm{~ms}$ for the entire sweep).

magnitude of allowable current and temperature fluctuations can be obtained with the knowledge that the laser frequency changes at the rate $-27.1 \mathrm{GHz} / \mathrm{K}$ and -3.2 $\mathrm{GHz} / \mathrm{mA}$. Hence for a typical locking bandwidth of 500 MHz, temperature fluctuations must not exceed a few millidegrees and the injection current must be stabilized at the microamp level. We have constructed temperature and current servos that achieve this level of stability. Their performance was verified by monitoring the frequency of a free-running laser (no optical feedback) using the cesium absorption line. The laser frequency was tuned to the steep edge of the Doppler-broadened line formed by transitions from the $6^{2} S_{1 / 2} F=4$ ground state of cesium. The absorption of laser light by the atomic vapor was recorded as the laser was allowed to run without adjustment. After calibrating the slope of this frequency discriminator we found that the laser center frequency experienced short term fluctuations of $\approx 50 \mathrm{MHz}$ superimposed on a long-term drift of $\approx 3 \mathrm{MHz} / \mathrm{min}$. For periods of less than $2 \mathrm{~h}_{\text {s }}$ these frequency excursions were well within the $500 \mathrm{MHz}$ locking bandwidth of the external-cavity laser described above.

In conjunction with the current and temperature stabilization, the confocal Fabry-Perot resonator was used as an optical feedback element to reduce the laser linewidth and provide a tuning mechanism. To determine the magnitude of linewidth reduction achieved, two external-cavity lasers were constructed in order to perform optical heterodyne detection. Figure 3 shows the beat note of the two lasers on an rf spectrum analyzer. The broad spectrum was obtained when the two lasers were not optically locked to their confocal Fabry-Perot cavities. When the two lasers were each locked to independent cavities, the narrow spectrum was obtained. The width of the beat note spectrum was reduced from $40 \mathrm{MHz}$ to $130 \mathrm{kHz}$. When optically locked, the laser linewidths were limited by the differential stability of the Fabry-Perot cavities of both lasers. (Locking to adjacent longitudinal modes of a single cavity gave a heterodyne signal of linewidth $50 \mathrm{kHz}$.)

Given the inherent lack of stability of most cavity

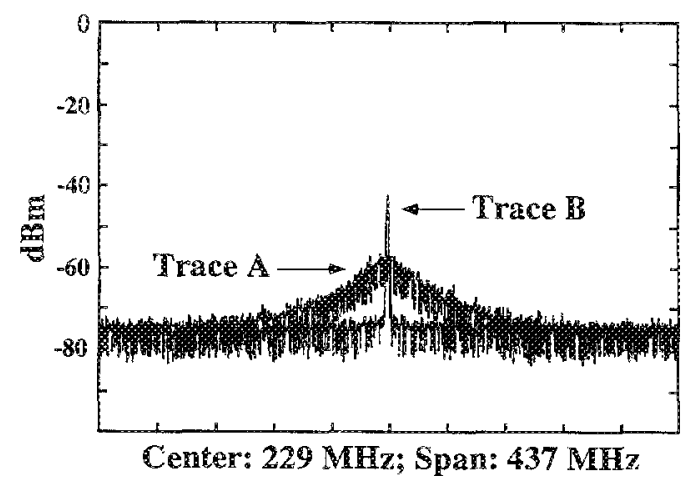

FIG. 3. Einewidth reduction measured by optical heterodyne technique. These two traces were taken with a rf spectrum analyzer and indicate a reduction in beatnote linewidth from $40 \mathrm{MHz}$ (trace A) to $130 \mathrm{kHz}$ (trace 13$)$.

structures, the laser was actively stabilized to an atomic resonance by mounting one of the mirrors on a piezoelectric translator (PZT) and constructing an automatic frequency control (AFC) loop. The frequency error signal was obtained as follows. Saturation spectroscopy was performed in order to resolve the cesium hyperfine lines. A small sinusoidal modulation was applied to the piezoelectric translator upon which $M_{2}$ was mounted. This gave rise

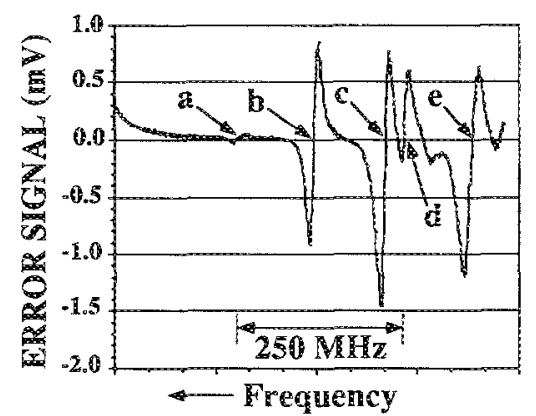

(a)

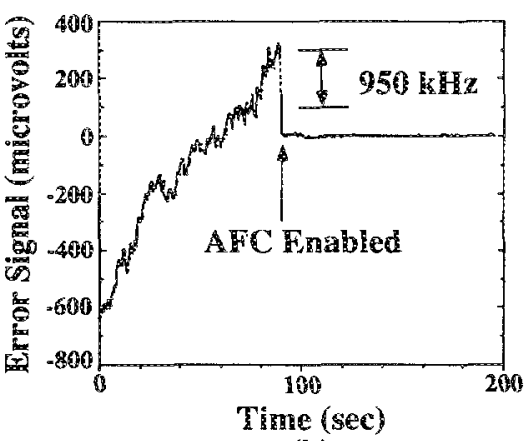

(b)

FIG. 4. (a) Open-loop frequency error signal (lock-in output, see Fig. 1) is recorded as the laser frequency is scanned across transitions from the cesium $6^{2} S_{1 / 2} F=4$ ground state. The labeled zero crossings correspond to the cesium principal resonances (pr) and crossover resonances (cr) observed in saturation spectroscopy. For crossover resonances, line center is halifway between the two levels given for $F$ : (a) pr $F=4 \rightarrow F=5$; (b) er $F=4 \rightarrow F^{\prime}=4,5$; (c) cr $F^{\prime}=4 \rightarrow F^{\prime}=3,5$; (d) pr $F=4 \rightarrow F^{\prime}=4$; (e) $\mathrm{cr} F=4 \rightarrow F^{x}=3,4$. The separation of (c) and (d) is $25 \mathrm{MHz}$. (b) The error signal is displayed under open and closed loop conditions. At $t=90$ $s$ the AFC loop is closed nulling the error signal. Residual fuctuations are $10 \mathrm{kHz}$ peak to peak. 
to a modulation of the laser frequency, and therefore, to the saturated absorption signal from photodetector $D_{1}$. Demodulation of the absorption signal with a lock-in amplifier produced a signed error signal that was proportional to the first derivative of the cesium spectrum. Figure 4 (a) shows the error signal as the laser was scanned across transitions from the $6^{2} S_{1 / 2} F=4$ ground state. The scan width was greater than the locking bandwidth so the laser's injection current was swept symchronously with the locking cavity. The opticaly locked laser was then tuned near the crossover resonance between the $6^{2} \widehat{S}_{1 / 2} F=4$ ground state and the $6^{2} P_{3 / 2} F=4$ and $F=5$ excited states. Figure $4(b)$ shows the effect upon the error signal of closing the frequency control loop. Once the slope of the error signal was calibrated, we found that the servo imited frequency excursions to $\pm 10 \mathrm{kHz}$ as measured inside the servo loop. Absolute stability with an independent reference was not invesigated. Although our discussion has centered on work near the cesium transition at $850 \mathrm{~nm}$, we have obtained similar results with AlGaAs lasers around the 780 nm transition of rubidium. This wavelength is significant because it represents the second harmonic of $1.56 \mu$, a wavelength utilized in fiber optic communication systems.

In conclusion, a hybrid optical/electronic stabilization technique has been demonstrated. Once the laser's temperature and injection current were reasonably well stabilized, optical feedback from a confocal Fabry-Perot cavity was used to reduce the laser's linewidth. Long-term frequency stability was achieved using an automatic frequency control loop incorporating the sub-Doppier spectrum of cesium vapor.

We would like to acknowledge the assistance of Leo Kollberg in setting up the optical feedback system. This work was funded by the Venture Research Unit of British Petrolium.

${ }^{1}$ M. Ohtsu, J. Lightwave Tcchnol. 6, 245 (1988).

${ }^{2}$ C. J. Nielsen and J. H. Osmundsen, Flectron. Lett. 19, 644 (1983).

${ }^{3} \mathrm{~S}$. Saito, O. Nisson, and $Y$. Yamamoto, IEEE J. Quantum Electron. (QE-18, 1712 (1982).

${ }^{4}$ M. Ito and T. Kimura, IEEE J. Quantum Electron. QE-16, 69 (1980)

5. Dahmani, L. Ilollberg, and R. Drullinger, Opt. Lett. L2, 876 (1987).

${ }^{6} \mathrm{H}$. Li and H. R. Telle, IEEE $I$. Quantum Electron. 25, 257 (1989).

${ }^{7}$ A. J. Wallard, J. Physics E. 5, 926 (1972). 